\title{
Surgery for Patients with Radiation-Induced Constrictive Pericarditis
}

\author{
Shixiong Wei, MD, Lin Zhang, MD, Huimin Cui, MD, Lianggang Li, MD, Tong Ren, MD, Shengli Jiang, PhD \\ Department of Cardiovascular Surgery, PLA General Hospital, Institute of Cardiac Surgery, Beijing, China
}

\section{ABSTRACT}

Radiation-induced heart disease (RIHD) is a major side effect of chest radiation therapy (RT). Most changes of pericardium will occur within a few weeks after receiving chest RT, while most of them will take decades or more to become constrictive pericarditis. Pericardiectomy is an effective treatment method. Here, we report 2 cases of radiation pericarditis after chest RT at our center.

\section{INTRODUCTION}

Cardiovascular diseases and malignant neoplasms are major causes of morbidity and death today. People have realized that cardiovascular diseases may occur in cancer survivors, who previously received chest radiation therapy (RT) [Bhattacharya 2016; Groarke 2014]. Radiation-induced heart disease (RIHD) is a major side effect of RT, including coronary artery diseases, pericarditis, valvular diseases, cardiomyopathy, abnormal cardiac conduction path, etc. [Stewart 2013; Cohn 1967]. The occurrence of these diseases is linked to the techniques used in RT, area of the heart exposed to RT, drug dosage, patient age, and their existing cardiovascular risk factors [Groarke 2014; Yusuf 2011].

Most changes of pericardium will occur within a few weeks after receiving chest RT, while many may take decades or more to develop into constrictive pericarditis [Bhattacharya 2016; Stewart 2013]. As therapeutic techniques improve, patient survival from malignant neoplasms also has been prolonged. However, RT still is one of many treatment methods, and patients with radiation pericarditis can show both subclinical symptoms and major cardiovascular adverse events [Cutter 2011]. So, clinicians should pay heed to this situation. Here, we report 2 cases of radiation pericarditis after chest RT.

\section{CASE REPORT}

Patient I: The first patient was a 41-year-old woman diagnosed with constrictive pericarditis. She came to our hospital with chest distress and shortness of breath. No tumor

Received April 10, 2019; accepted September 16, 2019.

Correspondence: Prof. Shengli Fiang, Department of Cardiovascular Surgery, PLA General Hospital, Institute of Cardiac Surgery, Beijing 100853, China (e-mail:jiangsl301@sina.com). cells were found in drained fluid, and a tuberculin test (PPD test) was negative. The preoperative central venous pressure (CVP) was $30 \mathrm{mmHg}$. She was given diuresis, anti-infection, thymosin, pericardium, and pleural injection of interleukin, however the patient's symptoms didn't significantly improve. Twenty years ago, this patient was diagnosed with lung cancer. After 3 times receiving chemotherapy with cyclophosphamide, vincristine, methotrexate, and etoposide (COMVP plan), she was given RT. After receiving RT with Co60 30 times, reexamination of this patient in 1994 showed that she had recovered. CT examination of admission revealed pericardium thickening, which is consistent with the characteristics of constrictive pericarditis (Figure 1). Hospitalized transthoracic echocardiography (TTE) revealed constrictive pericarditis, moderate mitral regurgitation, moderate tricuspid regurgitation, mild pulmonary hypertension $(52 \mathrm{mmHg})$, and occupying lesions in the right ventricular outflow tract. Left ventricular ejection fraction (LVEF) was $55 \%$. Abdominal ultrasound showed insignificant enlargement of the liver and spleen, which was considered a secondary change of constrictive pericarditis.

Patient II: This patient was a 52-year-old woman, who came to our hospital for treatment of palpitations, cough, chest tightness, and lower limb edema. Her PPD test was negative. No acid-fast bacilli and tumor cells were found in the pleural effusion culture and cytological examination. The preoperative CVP was $20 \mathrm{mmHg}$. TTE showed a large amount of pleural effusion. She was given diuretic and antituberculous drugs, but her symptoms did not significantly improve. Thirty years ago, this patient was diagnosed with Hodgkin's lymphoma. After she was given a course of chemotherapy, the patient received RT. Her Hodgkin's lymphoma was cured after one year of RT treatment. Preoperative TTE suggested her aortic valve was slightly calcified with a small amount of regurgitation; she also had a widened ascending aorta, moderate amount of left pleural effusion, and small amount of pericardium effusion. LVEF was $55 \%$. Abdominal ultrasound showed mild enlargement of liver and spleen, which was seen as a secondary change of constrictive pericarditis.

\section{RESULTS}

After hospitalization, both patients were given cardiotonic, diuretic, and nutritional support treatment. To avoid the influence of the patient's respiratory function during the perioperative period, a median thoracotomy was adopted. After the sternum is split, the pericardium was stripped up, down and on both sides by sharp and blunt separation under 


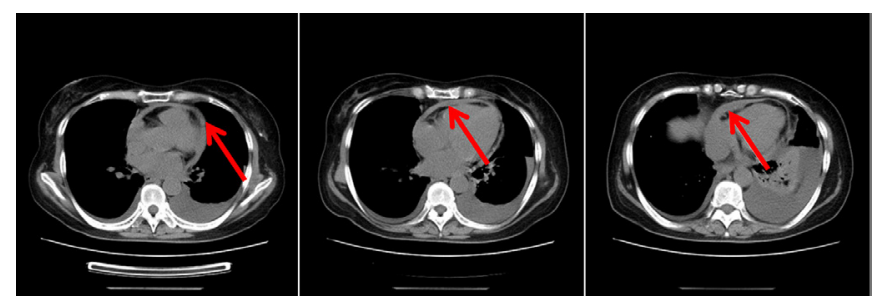

Figure 1. Preoperative CT scan showed pericardium was obviously thickened at the arrowhead, which conforms to the characteristics of constrictive pericarditis.
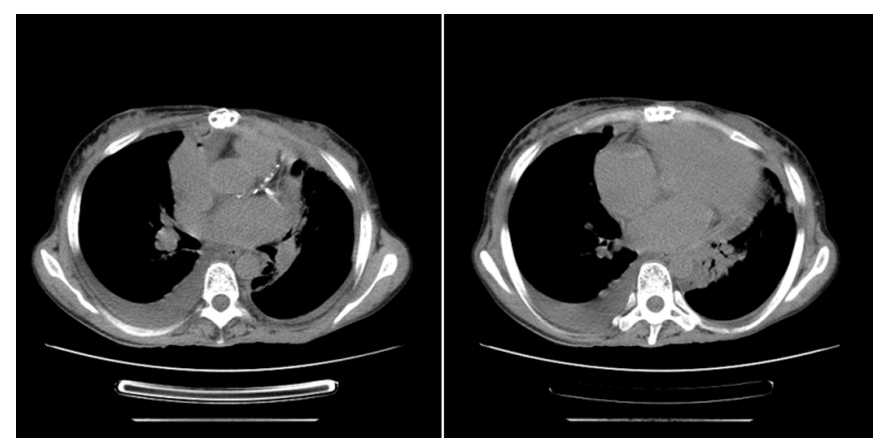

Figure 2. Postoperative CT scan showed normal pericardium thickness and no obvious calcification.

non-extracorporeal circulation. Our suggestion was that pericardium denudation should be performed as far as possible without extracorporeal circulation, but extracorporeal circulation should be applied when other heart valve operations are performed at the same time, or when the pericardium denudation situation is not satisfactory. Care should be taken to avoid damaging myocardial tissue, during the process. The separation should follow the sequence of left ventricular outflow tract, left ventricle, right ventricular outflow tract, right ventricle, and finally, superior and inferior vena cava. The stripping scope should be wide enough: The right side should exceed the atrioventricular groove, and the left side should strip the front and side of the left ventricle to reach the left phrenic nerve. Sufficient care should be taken when dissociating the interventricular sulcus to avoid damaging the anterior descending coronary artery. During the operation, we confirmed a significant thickening of the parietal pericardium. The anterior and side wall of the left ventricle, the pulmonary artery, and entrance to the superior and inferior vena cava obviously were adhered. We carefully separated and completely cut off the thickened pericardium (Figure 2). CVP decreased from 30 to $14 \mathrm{mmHg}$ and 20 to $5 \mathrm{mmHg}$, respectively. Results of immediate TTE showed good cardiac function.

Both patients smoothly recovered. No pericardium effusion was discovered, and no nosocomial infection or complications occurred. TTE was reexamined one week after operation and no abnormality was discovered. The pathological report of pericardium tissue suggested hyperplastic fibrous tissue with infiltration of hyalinization and chronic inflammatory cells (Figure 3).

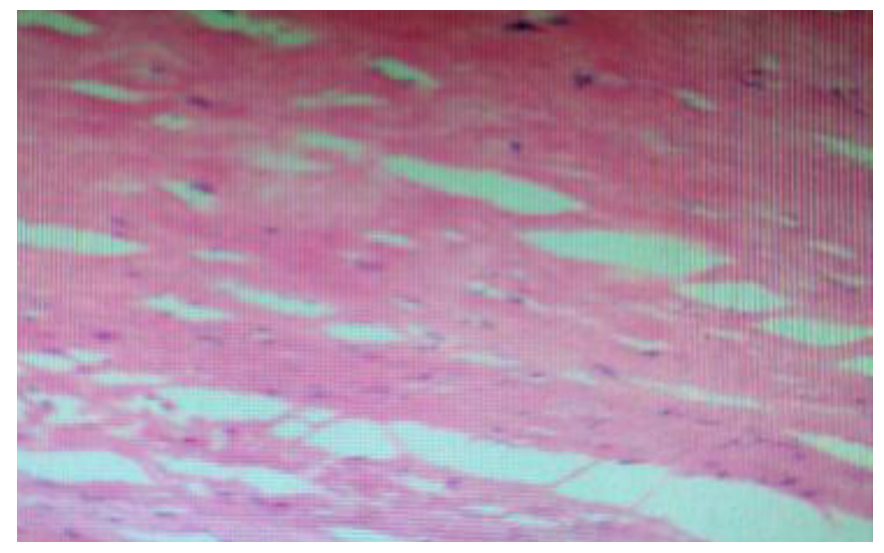

Figure 3. Pathological results showed hyperplastic fibrous tissue with hyalinization, chronic inflammatory cells, and local hemorrhage could be seen in pericardium tissue.

A year after discharge, we followed up with both patients. Patient I gradually developed chest tightness and dyspnea. Coronary angiography showed $70 \%$ blockage of the circumflex branch. TTE showed LVEF was 40\%. After placing a stent, the patient's symptoms improved. Patient II reported no symptoms since the operation, and she does not currently take any drugs.

\section{DIscussion}

Based on clinical symptoms, imaging findings, physical examination, and elevated CVP, the 2 patients were diagnosed with constrictive pericarditis. Tuberculous constrictive pericarditis could be excluded, according to results of the PPD test, pleural effusion culture, cytological examination, and postoperative pathology. Rheumatic and traumatic pericarditis also could be excluded, based on patient medical history and hematological exam results. Because a definite history of radiotherapy, they were diagnosed as radiation pericarditis [Curigliano 2012; NCCN 2012].

Compared with the 1970s, when acute pericarditis was more common after RT, modern medicine has reduced these complications because of improved RT technology and the reduction of radiation dose usage [Cohn 1967]. But $7 \%$ to $20 \%$ of patients receiving chest RT still suffer from chronic pericarditis after decades [Barbetakis 2010; Di Matteo 1978; Ling 1999]. Some scholars believe that radiation pericarditis occurs when the exposure area is larger than $30 \%$ of the heart area or the dose of RT drugs reaches 50 Gy [Madan 2015]. Myocardial ischemia and diffuse myocardial fibrosis caused by micro vascular injury are the pathological changes of radiation cardiomyopathy; this leads to a series of functional changes mainly due to impaired ventricular diastolic function [Darby 2010]. Clinical manifestations range from nonspecific symptoms to congestive heart failure. Radiation pericarditis often is accompanied by myocardial fibrosis (the right ventricle usually is severely damaged because its anatomical location is closed to the chest wall), coronary artery 
stenosis (Patient II developed coronary heart disease symptoms 1 year after pericardiectomy), valve disease, etc. So, it is reported that compared with constrictive pericarditis caused by rheumatoid disease, tuberculosis, tumor, trauma and other reasons, the survival rate of patients with radiation pericarditis is lower (7-year survival rate reported as being 27\%) [Ertog 2004]. Therefore, for patients diagnosed with constrictive pericarditis which may have been caused by RT, clinicians should conduct a comprehensive investigation of the patient's cardiovascular system.

The constrictive pericarditis can be screened by routine non-invasive imaging examination. Its imaging manifestations include pericardium thickening, inferior vena cava dilatation, pericardium and pleural effusion, tubular ventricles, and abdominal septum bounce. Generally, pericardium thickness of $4 \mathrm{~cm}$ on CT or MRI highly indicates constrictive pericarditis, but it should be noted that about $20 \%$ of patients with constrictive pericarditis confirmed by surgery do not have pericardium thickening [Yared 2010; Khandaker 2010; Napolitano 2009]. In addition, the increased E velocities peak of the septal compared with the lateral mitral valve annulus in TTE also is confirmed to specifically diagnose constrictive pericarditis. The phenomenon of ventricular septal bounce on TTE or MRI is deemed to be the most characteristic diagnostic basis for constrictive pericarditis, with a reported sensitivity on TTE of $62 \%$ to $81 \%$ and a specificity of $93 \%$ to $100 \%$ [Napolitano 2009]. The treatment of radiation pericarditis still is improving. The fibrosis caused by high-dose radiation makes the operation difficult, which is a question that all surgeons face. Veinot et al reported that the frequency of pericardium disease was as high as $70 \%$ when thoracic surgery was performed in patients with radiotherapy [Veinot 1996].

The European Society of Cardiology reported that the death rate of pericardium denudation was as high as $6 \%$ to $12 \%$. The main surgical complications are acute heart failure and heart rupture [Maisch 2004]. Busch et al reported that the decrease of LVEF and right ventricular dilation were independent predictors of early death, while coronary artery disease, chronic obstructive pulmonary disease and renal damage were predictors of late death [Busch 2015]. This was consistent with the fact that our patients did not have complications, such as heart failure, after surgery.

\section{CONCLUSION}

With the improvement of medical technology, more cancer patients are surviving. However, some patients' hearts are exposed to radiation during $\mathrm{RT}$, which increases the risk of cardiovascular complications. Moreover, due to the longterm delay between the time of exposure to radiation, the appearance of clinical symptoms and correct diagnosis, the report of related complications may be far from adequate.

At the same time, for patients who have received chest RT, it is necessary to conduct regular screening for cardiovascular diseases. Early detection of such complications can prompt intervention measures to improve cardiovascular prognosis. A multimodality approach of TTE with complementary CT or MRI can facilitate diagnosis of post-radiation constrictive pericarditis. Since the disease often occurs many years after the patient received RT, the follow-up period should be long enough [John 2014].

\section{REFERENCES}

Barbetakis N, Xenikakis T, Paliouras D, Asteriou C, Samanidis G, Kleontas A, Lafaras C, Platogiannis D, Bischiniotis T, Tsilikas C. 2010. Pericardiectomy for radiation-induced constrictive pericarditis. Hellenic J Cardiol 51:214-218.

Bhattacharya S, Asaithamby A. 2016. Ionizing radiation and heart risks. Semin Cell Dev Biol 58:14-25.

Busch C, Penov K, Amorim PA, Garbade J, Davierwala P, Schuler GC, et al. 2015. Risk factors for mortality after pericardiectomy for chronic constrictive pericarditis in a large single-centre cohort. Eur J Cardiothorac Surg. 48:110-6.

Cohn KE, Stewart JR, Fajardo LF, Hancock EW. 1967. Heart disease following radiation. Medicine (Baltimore) 46:281-298.

Curigliano G, Cardinale D, Suter T, Plataniotis G, de Azambuja E, Sandri MT, Criscitiello C, Goldhirsch A, Cipolla C, Roila F, Group EGW. 2012. Cardiovascular toxicity induced by chemotherapy, targeted agents and radiotherapy: ESMO Clinical Practice Guidelines. Ann Oncol 23(Suppl. 7):vii155-vii166.

Cutter DJ, Darby SC, Yusuf SW. 2011. Risks of heart disease after radiotherapy. Tex Heart Inst J 38:257-258.

Darby SC, Cutter DJ, Boerma M, Constine LS, Fajardo LF, Kodama K, Mabuchi K, Marks LB, Mettler FA, Pierce LJ, Trott KR, Yeh ET, Shore RE. 2010. Radiation-related heart disease: current knowledge and future prospects. Int J Radiat Oncol Biol Phys 76:656-665.

Di Matteo J, Vacheron A, Heulin A, Meeus L, Di Matteo G, Gilles R, Delage F, de Ratuld A. 1978. Cardiac complications of thoracic radiotherapy. Arch Mal Coeur Vaiss 71:447-452.

Ertog SC, Thambidorai SK, Parakh K, Schoenhagen P, Ozduran V, Houghtaling PL, Lytle BW, Blackstone EH, Lauer MS, Klein AL. 2004. Constrictive pericarditis: etiology and cause-specific survival after pericardiectomy. J Am Coll Cardiol 43:1445-1452.

Groarke JD, Nguyen PL, Nohria A, Ferrari R, Cheng S, Moslehi J. 2014. Cardiovascular complications of radiation therapy for thoracic malignancies: the role for non-invasive imaging for detection of cardiovascular disease. Eur Heart J 35 (10):612-23.

John D. Groarke, Paul L. Nguyen, Anju Nohria, et al. 2014. Cardiovascular complications of radiation therapy for thoracic malignancies: the role for non-invasive imaging for detection of cardiovascular disease. European Heart Journal 35, 612-623.

Khandaker MH, Espinosa RE, Nishimura RA, Sinak LJ, Hayes SN, Melduni RM, Oh JK. 2010. Pericardial disease: diagnosis and management. Mayo Clin Proc 85: 572-593.

Ling LH, Oh JK, Schaff HV, Danielson GK, Mahoney DW, Seward JB, Tajik AJ. 1999. Constrictive pericarditis in the modern era: evolving clinical spectrum and impact on outcome after pericardiectomy. Circulation 100:1380-1386.

Madan R, Benson R, Sharma D, et al. 2015. Radiation induced heart disease: pathogenesis, management and review literature. J Egypt Natl Canc Inst 27:187-93. 
Maisch B, Seferovi PM, Risti AD, Erbel R, Rienmüller R, Adler Y, et al. 2004. Guidelines on the Diagnosis and Management of Pericardial Diseases. Eur Heart J. 25:587-610.

Napolitano G, Pressacco J, Paquet E. 2009. Imaging features of constrictive pericarditis: beyond pericardial thickening. Can Assoc Radiol J 60:40-46.

National Comprehensive Cancer Network. 2012. Clinical Practice Guidelines in Oncology: Hodgkins Lymphoma Version 2.2012.

Stewart FA, Seemann I, Hoving S, Russell NS. 2013. Understanding radiation-induced cardiovascular damage and strategies for intervention. Clin Oncol (R Coll Radiol) 25(10):617-24.

Veinot JP, Edwards WD. 1996. Pathology of radiation-induced heart disease: a surgical and autopsy study of 27 cases. Hum Pathol. 27:766-73.

Yared K, Baggish AL, Picard MH, Hoffmann U, Hung J. 2010. Multimodality imaging of pericardial diseases. JACC Cardiovasc Imaging 3:650-660.

Yusuf SW, Sami S, Daher IN. 2011. Radiation-induced heart disease: a clinical update. Cardiol Res Pract 317659. 